\title{
Letter to Editor: Uncommon presentation of intracerebral hemorrhage in a young male
}

\section{Editor}

Intracerebral hemorrhage accounts for approximately $10 \%$ to 20 $\%$ of all strokes ${ }^{1}$ and it has a case-fatality at 1 month over $40 \%{ }^{2}$ It occurs when a blood vessel within the brain parenchyma ruptures. ${ }^{1}$ The incidence of intracerebral hemorrhage increased strongly with age, with persons aged 85 years and over having an almost ten-fold increase in yearly risk of intracerebral hemorrhage compared to persons aged 45 to 54 years. ${ }^{2}$ Nevertheless, stroke incidence in young adults has been rising worldwide possibly due to an increasing incidence of risk factors, ${ }^{3}$ and slightly higher prevalence of hemorrhagic strokes has been reported in young patients. ${ }^{3,4}$ Hemorrhagic strokes have a wide spectrum of clinical manifestations, though acute onset headache, vomiting and severe increases in blood pressure are the most prevalent signs and symptoms, that lead to localized neurological signs, developing in a few minutes. ${ }^{5}$ Usually hemorrhagic strokes are accompanied by loose of consciousness and occasionally epileptic seizures. ${ }^{5} \mathrm{~A}$ deterioration of clinical signs and symptoms often happens within $24-72 \mathrm{~h}$ with intracerebral hemorrhage. ${ }^{6}$ Here, we present a 17-year-old male with sudden onset of motor deficit while exercising, with no other remarkable symptom or sign. A left parietal lobar hemorrhage was confirmed by MRI and CT scan of the brain.

A 17-year-old-male, Senior High School (SHS) student totally healthy before, was brought to the Orthopedics OPD because he was doing his usual daily burpee exercise regime in the afternoon and while in plank position, had a sudden loss of power in his right arm, with inability to move it. He denied trauma or pain in the arm. He was evaluated in Orthopedics OPD and diagnosed as a traumatic musculoskeletal disorder with possible damage to the right brachial plexus, treatment with NSAIDs was imposed but the patient did not improve. Two days later, since the patient remains with inability to move the right arm, the relatives decided to request an internal medicine consultation. The patient was examined and a consultation with a Neurologist was requested. A new careful history taking and complete physical and neurological examination was performed. There was no personal or family history of any chronic disease. No use of any medication or illicit drugs. On direct questioning he described a mild generalized headache $(4 / 10)$ which started with the motor deficits but immediately relieves with paracetamol $500 \mathrm{mg}$. No other symptoms were reported, no loss of consciousness, no agitation, no seizures. The neurological examination revealed a patient fully conscious, alert and oriented to time, person and place; with normal mental status, who arrived walking to the consulting room. He had a right central facial paresis and right brachial flaccid plegia, predominantly distal $(0 / 5)$, Proximal (2/5). Hyperreflexia +3 in the right bicep and triceps reflexes. No involvement of other cranial nerves, no sensory deficits. Blood Pressure (BP) on arrival was 120/70 mmHg, and Heart Rate (HR) 73 x min, with no other remarkable signs on the physical examination. The patient was admitted and some tests were requested: Haemoglobin (Hb) $(13.9 \mathrm{~g} \mid \mathrm{dl})$, white blood cells $\left(5.6 \times 10^{9} \mathrm{~L}\right)$, neutrophil count $(45.8 \%)$, platelet $(242 \times 10 \% / \mathrm{L})$. Fasting blood sugar $(\mathrm{FBS}) 4.0$ $\mathrm{mmol} / \mathrm{L}$. Erythrocyte sedimentation rate $(19 \mathrm{~mm} / \mathrm{hr})$, hepatitis B and $\mathrm{C}$ and HIV were non-reactive. Clothing Profile: Prothrombin Time
Volume 10 Issue 6 - 2020

\author{
Gertrudis de los Angeles Hernandez \\ Gonzalez,' Edith Camejo Rodriguez,' Eric \\ Yao Amakpa ${ }^{3}$ \\ 'Department of Internal Medicine and Therapeutics, University \\ of Health and Allied Sciences, School of Medicine, Ho Teaching \\ Hospital, Ghana \\ ${ }^{2}$ Department of Internal Medicine and Therapeutics, University \\ of Health and Allied Sciences, School of Medicine, Ho Teaching \\ Hospital, Ghana \\ ${ }^{3}$ Department of Obstetrics and Gynecology, Ho Teaching \\ Hospital, Ghana
}

\begin{abstract}
Correspondence: Gertrudis de los Angeles Hernandez Gonzalez. Consultant Neurologist, Department of Internal Medicine and Therapeutics, University of Health and Allied Sciences, School of Medicine. Ho Teaching Hospital, PMB 3I. Ho, Volta Region, Ghana, Tel +233 240836I85,

Email gertruhenandez@gmail.com
\end{abstract}

Received: November II, 2020 | Published: December 17, 2020

(PT) $8.3 \mathrm{sec}$, International Normalized Ratio (INR) 0.64, Activated Partial Thromboplastin Time (APTT) $10.5 \mathrm{sec}$. Negative rheumatoid factor, Lipid Profile: Total Cholesterol $5.17 \mathrm{mmol} / \mathrm{L}$, Triglycerides, $0.66 \mathrm{mmol} / \mathrm{L}$, HDL Cholesterol $1.80 \mathrm{mmol} / \mathrm{L}$, LDL Cholesterol 2.07 mmol/L. Creatinine: 70.0, Liver Function Test (LFT) are within normal parameters. Hb electrophoresis AA, COVID-19 RT- PCR Test was negative.

Neuroimaging studies were also done: Brain MRI, which reported a well-defined, rounded, T1 heterogeneous, (central core of iso intensity to grey matter with a rim of hyperintensity), collection measuring $(2.5 \mathrm{TR} \times 2.5 \mathrm{AP} \times 2.5 \mathrm{CC}) \mathrm{cm}$ in the left parietal lobe (Figure 1). This collection 'blooms' on T2/ SWI imaging by suggesting early subacute haemorrhage (Figure 2). There was surrounding vasogenic oedema, No enhancement was seen post-gadolinium administration and does not 'restrict' on DWI/ADC imaging. On a complementary Brain CT Scan done 2 days later was described as a hyperdense collection (mean attenuation of 58HU) in the left parietal lobe with minimal surrounding oedema. No significant mass effect or shift of midline structures is seen. Findings in-keeping with early left parietal lobe subacute haemorrhage (Figure 3).

A Magnetic Resonance Angiogram (MRA) of the Cranial Vessels was also performed in order to rule out the presence of any vascular malformation (Arteriovenous Malformation, Aneurysm, Cavernous or Venous Angioma, etc.). The images show a (2.3CC x 1.2TR $\times 1.8 \mathrm{AP}$ ) well-defined T1/T2/FLAIR hyperintense lesion in the left centrum semiovale. The lesion has a hemosiderin ring and shows restricted diffusion with areas of blooming on T2- suggestive of subacute intracerebral bleed. No arteriovenous malformations seen. No vessel's narrowing, displacement, dilatation. No vascular abnormalities (Figure 4). 


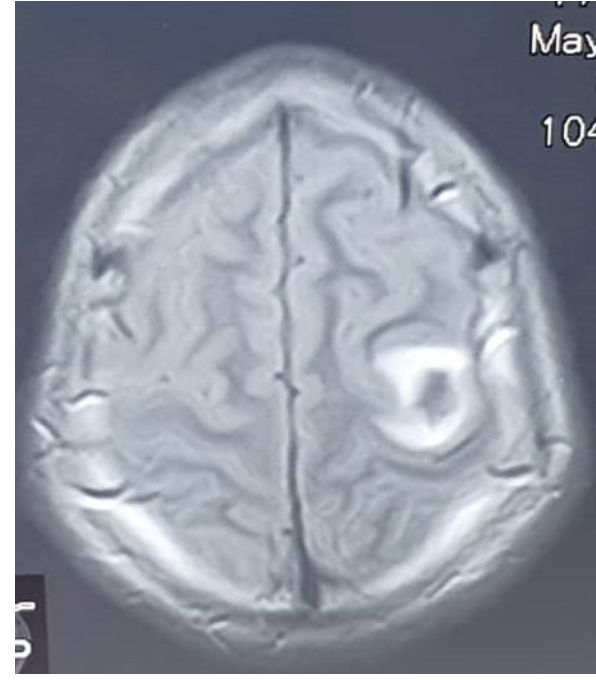

Figure I Brain MRITI-Left parietal central core of isointensity to grey matter with a rim of hyperintensity.

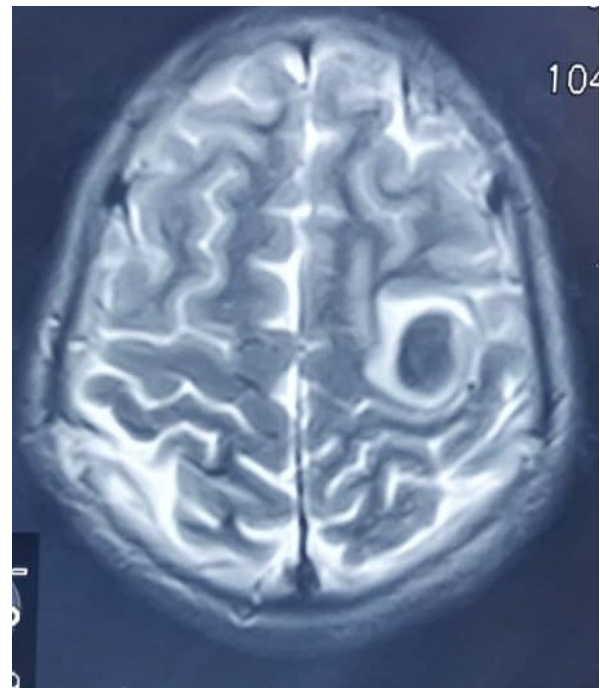

Figure 2 Brain MRIT2-Left parietal lobe collection "blooms" in T2.

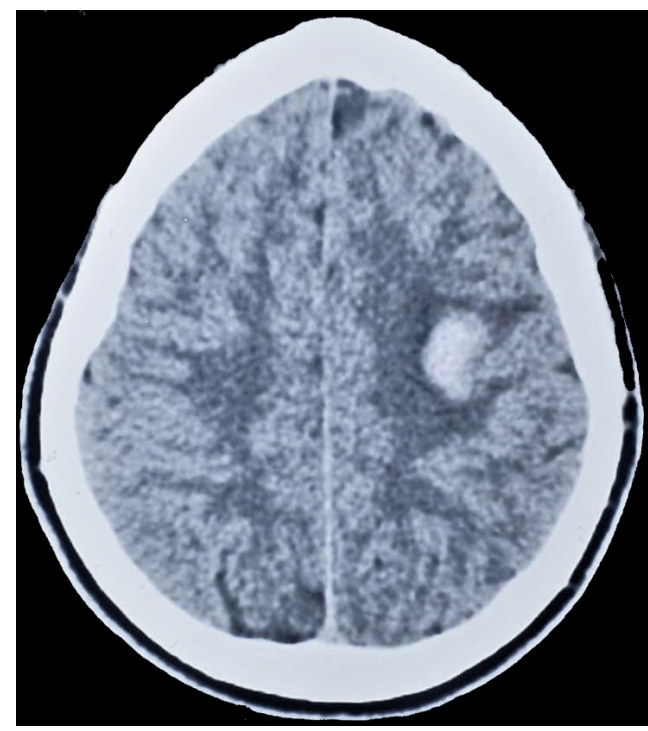

Figure 3 Brain CT scan-Left parietal hyperdense collection with minima surrounding edema.

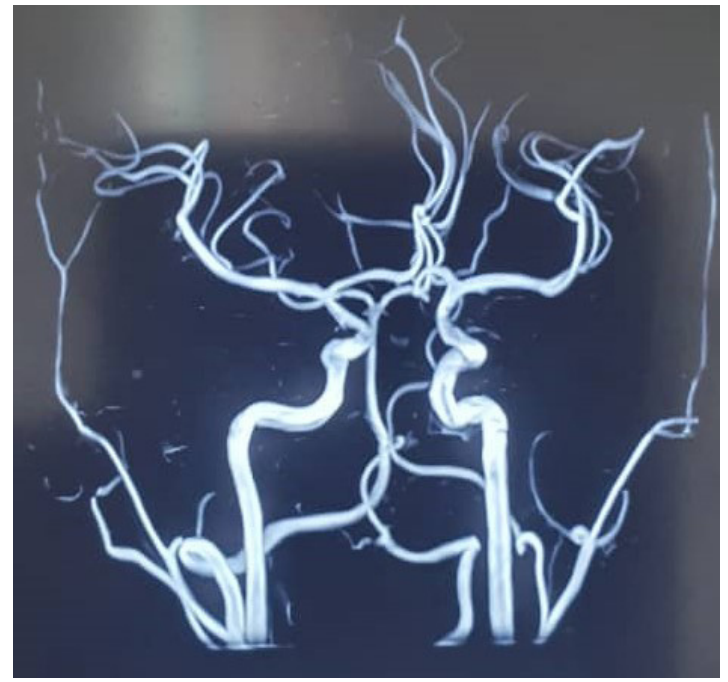

Figure 4 MRA of the cranial vessels-No vascular abnormalities.

The patient was comprehensively managed, involving symptomatic treatment and early rehabilitation. There were no complications and a remarkable neurological recovery occurred. He was discharged 8 days after admission and continued with physiotherapy. He was followed up in Neurology Consulting room weekly during the first month, and, monthly for 6 months. Three months after the onset of the condition he only had right brachial monoparesis $3 / 5$ distal in the hand, at 6 months only slight distal paresis in the right hand $4 / 5$. During the follow up of the patient there was no evidence of risk factors, predisposing conditions or structural abnormalities which could explain the left parietal ICH. The final diagnosis was Early-onset Cryptogenic Lobar Intracerebral Hemorrhage.

Stroke incidence in young adults has been rising worldwide possibly due to an increasing incidence of risk factors. In 2019 was proved that hemorrhagic stroke was slightly more common than ischemic stroke in young patients, evaluating stroke in young adults (16-55 years of age) in Taiwan, ${ }^{3}$ which has similar results than SIREN study in Stroke Among Young West Africans. ${ }^{4}$ In other studies the incidence of ICH in people aged 35 years has been estimated to be $0.3 / 100$ 000. The frequency of ICH among a series of stroke in young adults varies from $0.7 \%$ to $40 \%{ }^{7}$ The etiologic spectrum of ICH in young people may be wider than in older individuals and includes vascular malformations, hypertension, and drug use, although some studies report that 15-20 $\%$ of cases are consider cryptogenic. ${ }^{7}$ Cryptogenic ICH is defined by patients in whom there was no definite cause of ICH and is commonly located in the lobar region.

Intracerebral hemorrhage is clinically characterized by the abrupt onset, and rapid progression of the symptoms and signs in patients usually awake. Hemorrhagic strokes have a wide spectrum of clinical manifestations, the predominant symptoms for hemorrhagic stroke are acute headache and agitation, seizures with acute onset headache, vomiting and severe increases in blood pressure are the most prevalent signs and symptoms, that lead to localized neurological signs, developing in a few minutes. ${ }^{5}$ Usually hemorrhagic stroke are accompanied by loss of consciousness with a Glasgow Coma Scale (GCS score) of 8.97 (0.182), with minimum of 6 and maximum of $15 .{ }^{5}$ Early neurological deterioration of intracerebral hemorrhage has been recognized in many patients with hemorrhagic stroke that usually occurs within $24-72 \mathrm{~h}$ with intracerebral hemorrhage. ${ }^{6}$ Additionally, in the second to third weeks after the onset of intracerebral hemorrhage, 
many patients may undergo a deterioration of symptoms after the initial alleviation by conservative management, which indicates a subacute progression. ${ }^{8}$ Furthermore, the progression of hemorrhage may appear to be a chronic form, in which the gradual formation of an encapsulated intracerebral hematoma may cause progressive neurologic deficits over weeks or months. ${ }^{9}$

This case serves to remind physician the fact that ICHs may also appear in adolescents, and it may have several forms of clinical presentation, but is should be suspected if neurological focal signs appears abruptly indicating focal brain damage and the appropriate neuroimaging studies should be requested to confirm it since early recognition and treatment are crucial for favorable clinical outcomes and good functional recovery. It is also important to exhaust all possible investigation in order find out the possible etiology at this age, before diagnosing it as a Cryptogenic Early-Onset Intracerebral Hemorrhage.

\section{Acknowledgments}

None.

\section{Conflicts of interest}

The author declares no conflicts of interest.

\section{References}

1. Ikram MA, Wieberdink RG, Koudstaal PJ. International epidemiology of intracerebral hemorrhage. Current atherosclerosis reports. 2012;14(4):300-306.
2. Van Asch CJ, Luitse MJ, Rinkel GJ, et al. Incidence, case fatality, and functional outcome of intracerebral haemorrhage over time, according to age, sex, and ethnic origin: a systematic review and meta-analysis. The Lancet Neurology. 2010;9(2):167-176.

3. Chen CY, Lin P-T, Syu R-W, et al. Young Adult Stroke in Taiwan: etiologies and outcomes. 2019.

4. Sarfo FS, Ovbiagele B, Gebregziabher M, et al. Stroke among young west Africans: evidence from the SIREN (Stroke Investigative Research and Educational Network) large multisite case-control study. Stroke. 2018;49(5):1116-1122.

5. Ojaghihaghighi S, Vahdati SS, Mikaeilpour A, et al. Comparison of neurological clinical manifestation in patients with hemorrhagic and ischemic stroke. World J Emerg Med. 2017;8(1):34.

6. Chen S, Zeng L, Hu Z. Progressing haemorrhagic stroke: categories, causes, mechanisms and managements. J Neurol. 2014;261(11):20612078.

7. Ruíz-Sandoval JL, Cantu C, Barinagarrementeria F. Intracerebral hemorrhage in young people: analysis of risk factors, location, causes, and prognosis. Stroke. 1999;30(3):537-541.

8. Zazulia AR, Diringer MN, Derdeyn CP, et al. Progression of mass effect after intracerebral hemorrhage. Stroke. 1999;30(6):1167-1173.

9. Greiner-Perth R, Neubauer U, Schenke H. Chronic encapsulated intracerebral hematoma-a well-defined disease. Report on two cases and review of the literature. Neurosurgical review. 1997;20(4):231-238. 